\title{
3 Research Square

\section{Peripheral perfusion index predicting early hypoperfusion, reflecting lactate clearance rate and length of ICU stay in surgical patients: an observational study}

\author{
Xin ge Shi \\ Xinyang Central Hospital \\ Ming Xu \\ Xinyang Central Hospital \\ Xu Yu \\ Xinyang Central Hospital \\ Yibin Lu ( $D$ lyb_sxgicu@163.com ) \\ Xinyang Central Hospital
}

\section{Research article}

Keywords: peripheral perfusion index, surgical patients, critical patients, hypoperfusion, lactate clearance rate, length of ICU stay

Posted Date: February 11th, 2020

DOI: https://doi.org/10.21203/rs.2.23133/v1

License: (c) (i) This work is licensed under a Creative Commons Attribution 4.0 International License.

Read Full License 


\section{Abstract}

\section{Background}

Peripheral perfusion index (PPI) is associated with hypoperfusion in critical patients. Many researches focus on using PPI as a predictor in septic shock, but few studies report on its significance in surgical patients. We performed this study to find out association between PPI and surgical patients' prognoses.

\section{Methods}

It's a retrospective study from January to June 2019, on surgical patients transferred to ICU, Xinyang Central hospital, Henan province, China. The inclusive/exclusive criteria are followings, inclusive:1) age $\geq 18$ years old; 2 ) surgical length $\geq 120 \mathrm{~min}$; Exclusive: 1 ) died in ICU; 2) discharging against medical advice; 3 ) existing diseases affecting blood flow of upper limbs, for example, vascular thrombus in arms; 4) severe liver dysfunction Patients were grouped according to their length of ICU stay (LOS ICU, $>48 \mathrm{~h}$ regarded as prolonged ICU stay, otherwise non-prolonged). Baseline characteristics (age, gender, comorbidity), surgical sites and length, SOFA and APACHE II on 1st day in ICU, LOS ICU, mean artery pressure (MAP), lactate clearance rate (LCR), peripheral perfusion index (PPI), temperature (axillary) were collected and compared between the 2 groups. Correlation and ROC (receiver operating characteristic) analysis was also performed.

Results

Eventually, 72 patients were included, 30 in prolonged and 42 in non-prolonged group. Compared to nonprolong, patients in prolonged ICU stay group had higher lactate $6 \mathrm{~h}: 3.70 \pm 0.52 \mathrm{vs} 2.50 \pm 0.59 \mathrm{mmol} / \mathrm{L}$, p $<0.001 ; 12 \mathrm{~h}: 2.78 \pm 0.50$ vs $1.90 \pm 0.30 \mathrm{mmol} / \mathrm{L}, \mathrm{p}<0.001)$, lower LCR(0-6h: $-20 \%[-37 \%, 0 \%]$ vs $19 \%[9 \%$, $31 \%], p<0.001)$ and PPI (0h: $0.80 \pm 0.27$ vs $1.47 \pm 0.43, p<0.001 ; 6 h: 0.88 \pm 0.25$ vs $1.57 \pm 0.40, p<0.001)$. No difference was found in gender, age, surgical length, SOFA, APACHE II, temperature and comorbidity. Averaged $1 \mathrm{~h}$ and $6 \mathrm{~h}$ PPI were strongly associated with LCR $(0-6 \mathrm{~h}$, correlation coefficient $0.5521 \mathrm{~h}$ and $0.4366 \mathrm{~h}$ ) and LOS ICU(correlation coefficient: $-0.6391 \mathrm{~h}$ and $-0.5606 \mathrm{~h}$ ). The area under the receiver operating characteristic (AUC) of PPIT0 and PPIT1 for predicting prolonged ICU stay were 0.905 and 0.916 respectively. The cutoff values of PPIT0 and PPIT1 were 1.15 (with $73.8 \%$ sensitivity and $90.0 \%$ specificity) and 1.35 (with $83.3 \%$ sensitivity and $96.7 \%$ specificity).

\section{Conclusions}

PPI is associated with LOS ICU in surgical patients transferred to ICU. Lower PPI reflects slower lactate clearance rate and longer ICU stay.

\section{Backgrounds}

Many patients undergoing long time surgery will manifest increased level of blood lactate[1-3]. Hyperlactacidemia can cause disturbance of internal environment, then leading to increased 
complications and longer ICU stay, even death[1, 2, 4, 5]. For postoperative patients, we must ensure adequate perfusion in order to minimize the length of hyperglycemia and improve patients' outcomes. With regard to lactate, it provides information about prefusion, but not timely enough for its delay on reflecting hypoperfusion. And it's not real-time and non-invasive (requiring artery puncture).

Peripheral perfusion index (PPI) is an indicator reflecting hypoperfusion in critical patients[6]. It is measured using pulse co-oximetry technology which is characterized by being real-time and non-invasive. PPI is defined as "the ratio of pulsatile blood flow to the non-pulsatile blood flow", mirroring the strength of blood flow and quality of perfusion at sensor site, reflecting perfusion state of the body part[7, 8]. In contrast to lactate value, it's real-time and easy to monitor[8]. Besides, as a reflection of perfusion state, PPI may be a predictor of slower lactate clearance rate (LCR), indicating hypoperfusion in advance. Considering lower LCR is associated with longer ICU stay, we supposed that PPI might be related with length of ICU stay (LOS ICU). In order to prove our assumptions, this study was performed to find out the predictive significance of PPI in hypoperfusion and association with LOS ICU.

\section{Patients And Methods}

\section{Patients}

The Institutional Research and Ethics Committee of the Xinyang Central Hospital approved this study. Written informed consent was waived since it was a retrospective study.

This was a retrospective study of all surgical patients admitted to ICU in Xinyang Central Hospital (Henan province, China) from January 1st, 2019 to June 30th, 2019. The inclusive criteria are following:1) age $\geq$ 18 years old; 2) surgical length $\geq 120$ min. Exclusive criteria: 1 ) died in ICU; 2) discharging against medical advice; 3 ) existing diseases affecting blood flow of upper limbs, for example, vascular thrombus in arms; 4) severe liver dysfunction.

We defined patients whose LOS ICU > 48 h as "prolonged ICU stay", else as "non-prolonged ICU stay". After collecting data of enrolled patients, we divided them into two groups.

\section{Data collection}

The electronic medical records and anaesthesia charts of all recruited patients were examined to collect information on gender, age, comorbidity, surgical sites and length, SOFA and APACHE II on 1st day in ICU, LOS ICU, mean artery pressure (MAP), heart rate(HR), peripheral perfusion index (PPI), temperature (axillary).

Data were obtained continuously from the patient monitors (Philips IntelliVue MP50; Koninklijke Philips, The Netherlands) and automatically exported to the electronic medical records database. Experimental test results were also stored in it. PPI was captured by the pulse oximeter and blood pressure was measured using the oscillometric non-invasive technique or via radial arterial cannulation using pulse contour analysis. 
We collected MAP, HR and blood lactate on 3 different time points ( $0 \mathrm{~h}, 6 \mathrm{~h}, 12 \mathrm{~h}$ after admitted to ICU). As for PPI, we used its absolute value every $15 \mathrm{~min}$ to calculate the averaged value per hour. The averaged values of the 1st, 6 th and 12 th hour after ICU admission were calculated and collected.

Usually the axillary temperature is tested immediately after patients' admission to ICU, then every 6 hours afterwards. Lactate clearance rate $(\mathrm{LCR})$ was calculated by $\left(\mathrm{L}_{1}-\mathrm{L}_{2}\right) / \mathrm{T}_{1-2^{*}}$

Annotation: ${ }^{*} \mathrm{~L}_{1} / \mathrm{L}_{2}$ indicating lactate level at $\mathrm{T}_{1} / \mathrm{T}_{2}$ time point. $\mathrm{T}_{1-2}$ indicating interval between $\mathrm{T}_{1}$ and $\mathrm{T}_{2}$.

\section{Statistical analysis}

Descriptive analyses were performed for the non-prolonged and prolonged ICU stay groups. Continuous variables are expressed as means \pm standard deviations, and categorical variables are expressed as absolute values and percentages. For the continuous variables, the data were analyzed using Student's ttest, the Mann-Whitney $U$ test or the Kruskal-Wallis test depending on the data distribution and the number of variables. The categorical variables were analyzed using chi-square or Fisher's exact tests.

Correlation analyses were performed among PPI, lactate, lactate LCR and LOS ICU. to select spearman or pearson depends on the distribution of variables. Receiver operating characteristic (ROC)analyses were performed to test the significance of PPI to predict prolonged ICU stay.

All comparisons were two-tailed, and $\mathrm{P}<0.05$ was required to exclude the null hypothesis. The statistical analyses were performed using IBM SPSS Statistics, Version 20.0 (Armonk, NY: IBM Corp.).

\section{Results:}

36 patients were excluded for several reasons and 72 patients were recruited finally. They were divided into 2 groups according to their LOS ICU: 30 patients in "prolonged ICU stay" group, 42 patients in "nonprolonged ICU stay" group. The flow chart of patients' enrollment was showed in Fig. 1.

\section{Baseline characteristics}

There were no differences between the 2 groups in age, gender and the value of SOFA, APACHE II on the first day in ICU (Table 1). The surgical length in prolonged group was $220.97 \pm 60.79$ minutes while $197.74 \pm 55.31$ minutes in non-prolonged ICU stay group. We didn't see any differences on surgical length between the 2 groups. In addition to above parameters, we also analyzed the number of patients with comorbidities and undergoing different surgical types, none of any of them showed significant difference between the 2 groups. More details were displayed in the Table 1. 
Table 1

The baseline characteristics of enrolled patients

\begin{tabular}{|c|c|c|c|c|}
\hline & & $\begin{array}{l}\text { Prolonged ICU stay } \\
\mathrm{N}=30\end{array}$ & $\begin{array}{l}\text { Non-prolonged ICU stay } \\
\mathrm{N}=42\end{array}$ & $\mathbf{P}$ \\
\hline \multicolumn{2}{|l|}{ Age /year } & $55.40 \pm 10.44$ & $57.95 \pm 9.13$ & 0.274 \\
\hline \multicolumn{2}{|l|}{ Gender male/\% } & $19 / 63.33 \%$ & $25 / 59.52 \%$ & 0.744 \\
\hline \multicolumn{2}{|l|}{ LOS ICU /h } & $67.30 \pm 13.10$ & $34.19 \pm 8.18$ & $<0.001$ \\
\hline \multicolumn{2}{|l|}{ APACHE II } & $6.67 \pm 1.27$ & $6.41 \pm 1.36$ & 0.411 \\
\hline \multicolumn{2}{|l|}{ SOFA } & $5.47 \pm 1.43$ & $4.98 \pm 1.44$ & 0.158 \\
\hline \multicolumn{2}{|c|}{ Surgical length / min } & $220.97 \pm 60.79$ & $197.74 \pm 55.31$ & 0.096 \\
\hline \multirow[t]{7}{*}{ Comorbidity } & CAD & 9 & 11 & 0.722 \\
\hline & COPD & 5 & 6 & 1.000 \\
\hline & CKD & 3 & 4 & 1.000 \\
\hline & HTN & 11 & 13 & 0.612 \\
\hline & Diabetes & 8 & 9 & 0.606 \\
\hline & PMI & 2 & 4 & 1.000 \\
\hline & Others & 1 & 1 & 1.000 \\
\hline \multirow[t]{6}{*}{ Operative types } & Abdominal & 10 & 17 & 0.537 \\
\hline & Chest & 2 & 7 & 0.288 \\
\hline & Lung & 4 & 3 & 0.440 \\
\hline & Esophagus & 6 & 5 & 0.508 \\
\hline & GO & 5 & 5 & 1.000 \\
\hline & GA & 3 & 5 & 0.732 \\
\hline \multicolumn{5}{|c|}{$\begin{array}{l}\text { LOS ICU: length of ICU stay; APACHE II: Acute Physiology and Chronic Health Evaluation; SOFA: } \\
\text { Sequential Organ Failure Assessment; CAD: coronary artery disease; COPD: chronic obstructive } \\
\text { pulmonary disease; CKD: chronic kidney disease; HTN: hypertension; PMI: pacemaker implantation; } \\
\text { GO: gynecological oncology; GA: great artery. }\end{array}$} \\
\hline
\end{tabular}

\section{Hemodynamics and perfusion}

$\mathrm{HR}$, MAP, lactate, PPI and axillary temperate were collected at 3 different time points, $\mathrm{T}_{0}$ (on ICU admission), $\mathrm{T}_{1}\left(6 \mathrm{~h}\right.$ after admission), $\mathrm{T}_{2}$ (12 $\mathrm{h}$ after admission). 
Compare to non-prolonged ICU stay, prolonged group had lower PPI at $T_{0}(0.80 \pm 0.27$ vs $1.47 \pm 0.43, P<$ $0.001)$ and $T_{1}(0.88 \pm 0.25$ vs $1.57 \pm 0.40, P<0.001)$. For lactate, no differences at $T_{0}$, but prolonged group had significantly higher value than non-prolonged at $T_{1}(3.70 \pm 0.52$ vs $2.50 \pm 0.59 \mathrm{mmol} / \mathrm{L}, \mathrm{P}<0.001)$ and $\mathrm{T}_{2}(2.78 \pm 0.50$ vs $1.90 \pm 0.30 \mathrm{mmol} / \mathrm{L}, \mathrm{P}<0.001)$. Most patients in "prolonged ICU stay group" showed increased tendency on lactate, which led to minus LCR in $\mathrm{T}_{0-1}$ of this group $(-20 \%[-37 \%, 0 \%])$. LCR of non-prolonged group in $\mathrm{T}_{0-1}$ was $19 \%[9 \%, 31 \%]$, and $\mathrm{P}<0.001$. No difference of LCR in $\mathrm{T}_{1-2}$ was displayed between the 2 groups. More information was presented in Table 2.

Table 2

Comparison of hemodynamic and perfusion parameters between the two groups

\begin{tabular}{|c|c|c|c|c|}
\hline & & $\begin{array}{l}\text { Prolonged ICU stay } \\
\mathrm{N}=30\end{array}$ & $\begin{array}{l}\text { Non-prolonged ICU stay } \\
\mathrm{N}=42\end{array}$ & $\mathbf{P}$ \\
\hline \multirow[t]{3}{*}{ HR bpm } & T0 & $86.77 \pm 14.46$ & $86.69 \pm 12.11$ & 0.981 \\
\hline & T1 & $85.20 \pm 11.26$ & $88.41 \pm 10.16$ & 0.211 \\
\hline & T2 & $86.57 \pm 10.31$ & $90.00 \pm 8.25$ & 0.121 \\
\hline \multirow{3}{*}{$\begin{array}{l}\mathrm{MAP} \\
/ \mathrm{mmHg}\end{array}$} & TO & $74.03 \pm 6.43$ & $74.88 \pm 8.06$ & 0.622 \\
\hline & T1 & $75.33 \pm 4.44$ & $75.83 \pm 5.33$ & 0.667 \\
\hline & T2 & $75.53 \pm 5.86$ & $74.43 \pm 5.70$ & 0.425 \\
\hline \multirow{3}{*}{$\begin{array}{l}\text { Lactate } \\
\mathrm{mmol} / \mathrm{L}\end{array}$} & T0 & $3.19 \pm 0.71$ & $3.09 \pm 0.73$ & 0.544 \\
\hline & T1 & $3.70 \pm 0.52$ & $2.50 \pm 0.59$ & $<0.001$ \\
\hline & T2 & $2.78 \pm 0.50$ & $1.90 \pm 0.30$ & $<0.001$ \\
\hline \multirow{2}{*}{$\begin{array}{l}\mathrm{LCR} \\
/ \%\end{array}$} & $\mathrm{~T}_{0-1}$ & $-20 \%[-37 \%, 0 \%]$ & $19 \%[9 \%, 31 \%]$ & $<0.001$ \\
\hline & $\mathrm{T}_{1-2}$ & $25 \%$ [21\%, 32\%] & $20 \%[15 \%, 31 \%]$ & 0.339 \\
\hline \multirow[t]{3}{*}{ PPI } & T0 & $0.80 \pm 0.27$ & $1.47 \pm 0.43$ & $<0.001$ \\
\hline & T1 & $0.88 \pm 0.25$ & $1.57 \pm 0.40$ & $<0.001$ \\
\hline & T2 & $1.23 \pm 0.28$ & $1.22 \pm 0.34$ & 0.970 \\
\hline \multirow{3}{*}{${ }^{\mathrm{T}}{ }^{\circ} \mathrm{C}$} & TO & $36.39 \pm 0.32$ & $36.40 \pm 0.35$ & 0.893 \\
\hline & T1 & $36.84 \pm 0.20$ & $36.77 \pm 0.20$ & 0.168 \\
\hline & T2 & $37.01 \pm 0.41$ & $36.95 \pm 0.42$ & 0.530 \\
\hline
\end{tabular}

HR: heart rate; MAP: mean artery pressure; LCR: lactate clearance rate; PPI: peripheral perfusion index; T: temperature. 
Table 3

The correlation analyzations among perfusion and prognostic parameters

\begin{tabular}{|c|c|c|c|c|c|c|}
\hline & Lactate $T_{0}$ & Lactate $\mathrm{T}_{1}$ & Lactate $\mathrm{T}_{2}$ & $\operatorname{LCR}_{0-1}$ & LCR T $_{1-2}$ & LOS ICU \\
\hline $\mathrm{PPI} \mathrm{T}_{0}$ & 0.030 & $-0.561^{\star \star}$ & $-0.518^{\star \star}$ & $0.552^{\star \star}$ & -0.106 & $-0.639 * \star$ \\
\hline $\mathrm{PPI} \mathrm{T}_{1}$ & -0.063 & $-0.539 * \star$ & $-0.544^{\star \star}$ & $0.436^{\star *}$ & -0.119 & -0.560 ** \\
\hline $\mathrm{PPI} \mathrm{T}_{2}$ & 0.034 & 0.095 & -0.023 & -0.036 & 0.070 & 0.053 \\
\hline
\end{tabular}

\section{Correlation analyses}

By performing correlation analyzation, no association between lactate at T0 and $\mathrm{PPI}$ at any time point was found. As time went by, significantly negative associations were showed between lactate at $T_{1}$ and PPI at $\mathrm{T}_{1}$ (correlation coefficient (CC): $-0.561, \mathrm{P}<0.01$ ), $\mathrm{T}_{1}(\mathrm{CC}:-0.539, \mathrm{P}<0.01)$. At $\mathrm{T}_{2}$, lactate still had negative association with PPI at $\mathrm{T}_{0}(\mathrm{CC}:-0.518, \mathrm{P}<0.01), \mathrm{T}_{1}(\mathrm{CC}:-0.544, \mathrm{P}<0.01)$.

Lactate clearance rate in the first 6 hours in ICU (LCR $\left.T_{0-1}\right)$ was positively associated with PPI at $T_{0}$ (CC: $0.552, \mathrm{P}<0.01)$ and $\mathrm{PPI}$ at $\mathrm{T}_{1}(\mathrm{CC}: 0.436, \mathrm{P}<0.01)$. Besides relations with perfusion parameters, lower $\mathrm{PPI}$ (CC: $T_{1} / T_{2},-0.639 /-0.560, P<0.01$ ) was associated with longer LOS ICU.

In Figs. 2, 3, 4 and 5, we showed the tendency of LCR and LOS ICU when PPI increased. It's obvious to recognize the growing trend of LCR in $T_{0-1}$ if PPI $\left(T_{0}, T_{1}\right)$ increases (Fig. 2, Fig. 3). Besides, in Figs. 4 and 5 , LOS ICU decreased as PPI $\left(T_{0}, T_{1}\right)$ increased.

\section{ROC analyses}

ROC curves were drawn to compare the predictive values of PPI $\left(T_{0}, T_{1}\right)$ for non-prolonged ICU stay (Fig. 6). The AUC demonstrated that the predictive values of $\mathrm{PPI}_{\mathrm{T} 0}$ and $\mathrm{PPI}_{\mathrm{T} 1}$ were $0.905(95 \% \mathrm{Cl}: 0.836-$ 0.973), 0.916 (95\% Cl:0.850-0.982), respectively (Table 4). The cutoff values for $\mathrm{PPI}_{\mathrm{T} 0}, \mathrm{PPI}_{\mathrm{T} 1}$ were 1.15(sensitivity: 73.8\%; specificity: 90\%), 1.35 (sensitivity: 83.3\%; specificity: $96.7 \%$ ), respectively, based on the maximum Youden index.

Table 4

The results of ROC analysis for PPI $T_{0}$ and PPI $T_{1}$

\begin{tabular}{|llllll|}
\hline Variables & ROC area & $95 \% \mathrm{Cl}$ & Cutoff value & Sensitivity & Specificity \\
\hline PPI T 0 & 0.905 & $0.836-0.973$ & 1.15 & 0.738 & 0.900 \\
PPI T $_{1}$ & 0.916 & $0.850-0.982$ & 1.35 & 0.833 & 0.967 \\
\hline
\end{tabular}




\section{Discussion}

The most important finding of this research is PPI can predict hypotension happened on surgical patients in the first 1 hour and is associated with length of ICU stay. If PPI can reach 1.15 in the first hour in ICU, it predicts patients could discharge from ICU within $48 \mathrm{~h}$, with $73.8 \%$ sensitivity and $90.0 \%$ specificity.

Patients undergoing long time surgeries tend to manifest high level of lactate. This is a multifactorial phenomenon. Slow lactate clearance rate, inadequate perfusion, massive blood transfusion and application of anaesthetic drugs, all of these can lead to increased serum lactate after surgery[9-11]. It is known to us all that hyperlactacidemia is a strong indictor of poor prognosis[12, 13], because it will deteriorate internal environment and lead to multiple organ dysfunction[4]. We should minimize the length of hyperlactacidemia as much as possible. In regard to surgical patients, especially who are transferred to ICU after surgeries, going through big operations is a challenge. On top of that, they are at risk of increased blood lactate[1-3]. So, it is essential to ensure adequate perfusion to accelerate lactate clearance and shorten the length of hyperlactacidemia. If we can recognize hypotension in the early phase, we can take measures to improve perfusion more quickly and the shorten length of hyperlactacidemia.

We need indicators to guide clinic interventions to ensure perfusion. Despite that a lot of indexes have been proved useful in directing treatment, many of them are invasive or not real-time[14], which would weaken their significance in guiding therapies or predicting prognoses in the early phase. Compared to other indicators that are used in guiding shock treatment, PPI is non-invasive and real-time. Studies on PPI have confirmed its significance on reflecting tissue perfusion in critically ill patients[6, 15, 16].

Based on many previous researches, we carried out this retrospective study and confirmed that in the first hour in ICU, PPI has its role in predicting whether the patients would discharge within 48 hours or not, with a reasonable accuracy ( $73.8 \%$ sensitivity and $90.0 \%$ specificity). If we look forward, after 6 hours in ICU, for patients with $\mathrm{PPI} \geq 1.35$,they have $96.7 \%$ possibility of discharging from in the first 2 days. As time went by, after 12 hours, PPI of both groups could reach $1.2(1.23 \pm 0.28$ vs $1.22 \pm 0.34$ ), a value indicating an acceptable regional prefusion. No association was found between PPI $\mathrm{T}_{1}$ and LOS ICU.

Lactate and LCR are widely regarded as prognostic indexes for shock patients[2, 4, 12, 13]. Although in this study, both lactate $\left(T_{1}, T_{2}\right)$ and $\operatorname{LCR}\left(T_{0-1}\right)$ still have their significances, compared to PPI, which could be used to predict outcomes in the first hour in ICU, their shortcomings are obvious, not to mention that they're invasive and not real-time.

It's known to us all that at the initial period of hypoperfusion, peripheral vessels contract in order to supply enough blood returning to heart $[17,18]$. In this phase, macro vital signs, like HR and BP, are normal. Because macro circulation is stable, lactate doesn't increase. But PPI, which reflects regional perfusion, will decrease for the vessels contraction. This makes PPI superior to other macro parameters in indicating potential hypoperfusion. 
Different to other studies, our research not only proved PPI's predictive role in hypoperfusion, but also found its association with length of ICU stay. These findings help physicians to take measures more quickly, which may be helpful in improving patients' outcomes.

Low temperature and vascular diseases are the two main confounding factors which could affect value of PPI[19]. In this study, we excluded all patients with diseases affecting blood flow of upper limbs. In our routine nursing, we give warm-keeping service to all the patients, for example covering blankets. If necessary, we provide warm blower to keep patients warm. Besides, by comparing temperature between the 2 groups, no significant difference was found. Above all could exclude the confounding effects on PPI.

\section{Limitations}

This study has several limitations. First, its sample size is small. More studies on PPI with bigger sample size should be carried out in the future. Second, patients who died in ICU or discharged against medical advice were excluded. It would cause the included patients less severe. As we can see from the results, most patients' PPI could reach to 1.0 within 12 hours. Besides, the highest averaged lactate of both groups was less than $4.0 \mathrm{mmol} / \mathrm{L}$. As for vital signs of both groups, HR and MAP were also within normal range. These phenomena indicate patients recruited in the study were not so serious. It's better to confine our conclusions to surgical patients who are not in life-threatening conditions. As for patients on the edge of death, predicting their LOS ICU is much more complicated. Third, studying period is only 12 hours. The reason why we focus on a short period is that we want to find out predictive indicators in early clinic phase. It's worth to note that many complications could appear in the late stage, these complications may postpone the discharge. A previous study had confirmed PPI alteration was associated with development of severe complications[20]. So we have confidence that decreased PPI is associated with prolonged ICU stay even in the long term.

\section{Conclusions}

PPI can predict hypoperfusion at early stage in surgical patients transferred to ICU. Lower PPIs in first and sixth hours in ICU indicate prolonged ICU stay.

\section{Abbreviations}

PPI: peripheral perfusion index; SOFA: Sequential Organ Failure Assessment; APACHE II: Acute Physiology and Chronic Health Evaluation; HR: heart rate; MAP: mean artery pressure; LOS ICU: length of ICU stay; LCR: lactate clearance rate; $\mathrm{T}$ : temperature; Cl: confidence interval ; CC: correlation coefficient; ROC: receiver operating characteristic; AUC: receiver operating characteristic; CAD: coronary artery disease; COPD: chronic obstructive pulmonary disease; CKD: chronic kidney disease; HTN: hypertension; PMI: pacemaker implantation; GO: gynecological oncology; GA: great artery. 


\section{Declarations}

\section{Ethics approval and consent to participate}

The Institutional Research and Ethics Committee of the Xinyang Central Hospital approved this study for human subjects. Because this retrospective study only collected clinical data, the Institutional Research and Ethics Committee waived the need to obtain consent.

\section{Consent for publication}

Not applicable

\section{Availability of data and materials}

The datasets generated and/or analyzed during the current study are not publicly available due the security of our patients, but are available from the corresponding author on reasonable request

\section{Competing interests}

The authors declare that they have no competing interests

\section{Funding}

Not applicable

\section{Authors' contributions}

BL conceived and designed the study, interpreted data and helped draft the manuscript. XS participated in the study conception and design, recruited patients, collected data, performed the statistical analysis, interpreted the data and drafted the manuscript. MX and XY participated in technically support and data collection. All authors read and approved the final manuscript.

\section{Acknowledgements}

Not applicable

\section{References}

1. Velickovic J, Palibrk I, Milicic B, Velickovic D, Jovanovic B, Rakic G, Petrovic M, Bumbasirevic V: The association of early postoperative lactate levels with morbidity after elective major abdominal surgery. Bosnian journal of basic medical sciences 2019, 19(1):72-80.

2. Lemke M, Karanicolas PJ, Habashi R, Behman R, Coburn NG, Hanna SS, Law CHL, Hallet J: Elevated Lactate is Independently Associated with Adverse Outcomes Following Hepatectomy. World journal of surgery 2017, 41(12):3180-3188. 
3. Kanazawa T, Egi M, Shimizu K, Toda Y, Iwasaki T, Morimatsu H: Intraoperative change of lactate level is associated with postoperative outcomes in pediatric cardiac surgery patients: retrospective observational study. BMC anesthesiology 2015, 15:29.

4. Zhang Z, Xu X: Lactate clearance is a useful biomarker for the prediction of all-cause mortality in critically ill patients: a systematic review and meta-analysis*. Critical care medicine 2014, 42(9):2118-2125.

5. Mizock BA, Falk JL: Lactic acidosis in critical illness. Critical care medicine 1992, 20(1):80-93.

6. Sivaprasath P, Mookka Gounder R, Mythili B: Prediction of Shock by Peripheral Perfusion Index. Indian journal of pediatrics 2019, 86(10):903-908.

7. van Genderen ME, van Bommel J, Lima A: Monitoring peripheral perfusion in critically ill patients at the bedside. Current opinion in critical care 2012, 18(3):273-279.

8. Tafner P, Chen FK, Rabello RF, Correa TD, Chaves RCF, Serpa AN: Recent advances in bedside microcirculation assessment in critically ill patients. Revista Brasileira de terapia intensiva 2017, 29(2):238-247.

9. Maillet JM, Le Besnerais P, Cantoni M, Nataf P, Ruffenach A, Lessana A, Brodaty D: Frequency, risk factors, and outcome of hyperlactatemia after cardiac surgery. Chest 2003, 123(5):1361-1366.

10. Demers P, Elkouri S, Martineau R, Couturier A, Cartier R: Outcome with high blood lactate levels during cardiopulmonary bypass in adult cardiac operation. The Annals of thoracic surgery 2000, 70(6):2082-2086.

11. Kitterer D, Schwab M, Alscher MD, Braun N, Latus J: Drug-induced acid-base disorders. Pediatric nephrology (Berlin, Germany) 2015, 30(9):1407-1423.

12. Haas SA, Lange T, Saugel B, Petzoldt M, Fuhrmann V, Metschke M, Kluge S: Severe hyperlactatemia, lactate clearance and mortality in unselected critically ill patients. Intensive care medicine 2016, 42(2):202-210.

13. Rishu AH, Khan R, Al-Dorzi HM, Tamim HM, Al-Qahtani S, Al-Ghamdi G, Arabi YM: Even mild hyperlactatemia is associated with increased mortality in critically ill patients. Critical care (London, England) 2013, 17(5):R197.

14. Rhodes A, Evans LE, Alhazzani W, Levy MM, Antonelli M, Ferrer R, Kumar A, Sevransky JE, Sprung CL, Nunnally ME et al: Surviving Sepsis Campaign: International Guidelines for Management of Sepsis and Septic Shock: 2016. Intensive care medicine 2017, 43(3):304-377.

15. Duggappa DR, Lokesh M, Dixit A, Paul R, Raghavendra Rao RS, Prabha P: Perfusion index as a predictor of hypotension following spinal anaesthesia in lower segment caesarean section. Indian journal of anaesthesia 2017, 61(8):649-654.

16. He HW, Liu DW, Long Y, Wang XT: The peripheral perfusion index and transcutaneous oxygen challenge test are predictive of mortality in septic patients after resuscitation. Critical care (London, England) 2013, 17(3):R116.

17. Vincent JL, Ince C, Bakker J: Clinical review: Circulatory shock-an update: a tribute to Professor Max Harry Weil. Critical care (London, England) 2012, 16(6):239. 
18. Vincent JL, De Backer D: Circulatory shock. The New England journal of medicine 2013, 369(18):1726-1734.

19. Lima AP, Beelen $P$, Bakker J: Use of a peripheral perfusion index derived from the pulse oximetry signal as a noninvasive indicator of perfusion. Critical care medicine 2002, 30(6):1210-1213.

20. van Genderen ME, Paauwe J, de Jonge J, van der Valk RJ, Lima A, Bakker J, van Bommel J: Clinical assessment of peripheral perfusion to predict postoperative complications after major abdominal surgery early: a prospective observational study in adults. Critical care (London, England) 2014, 18(3):R114.

\section{Figures}




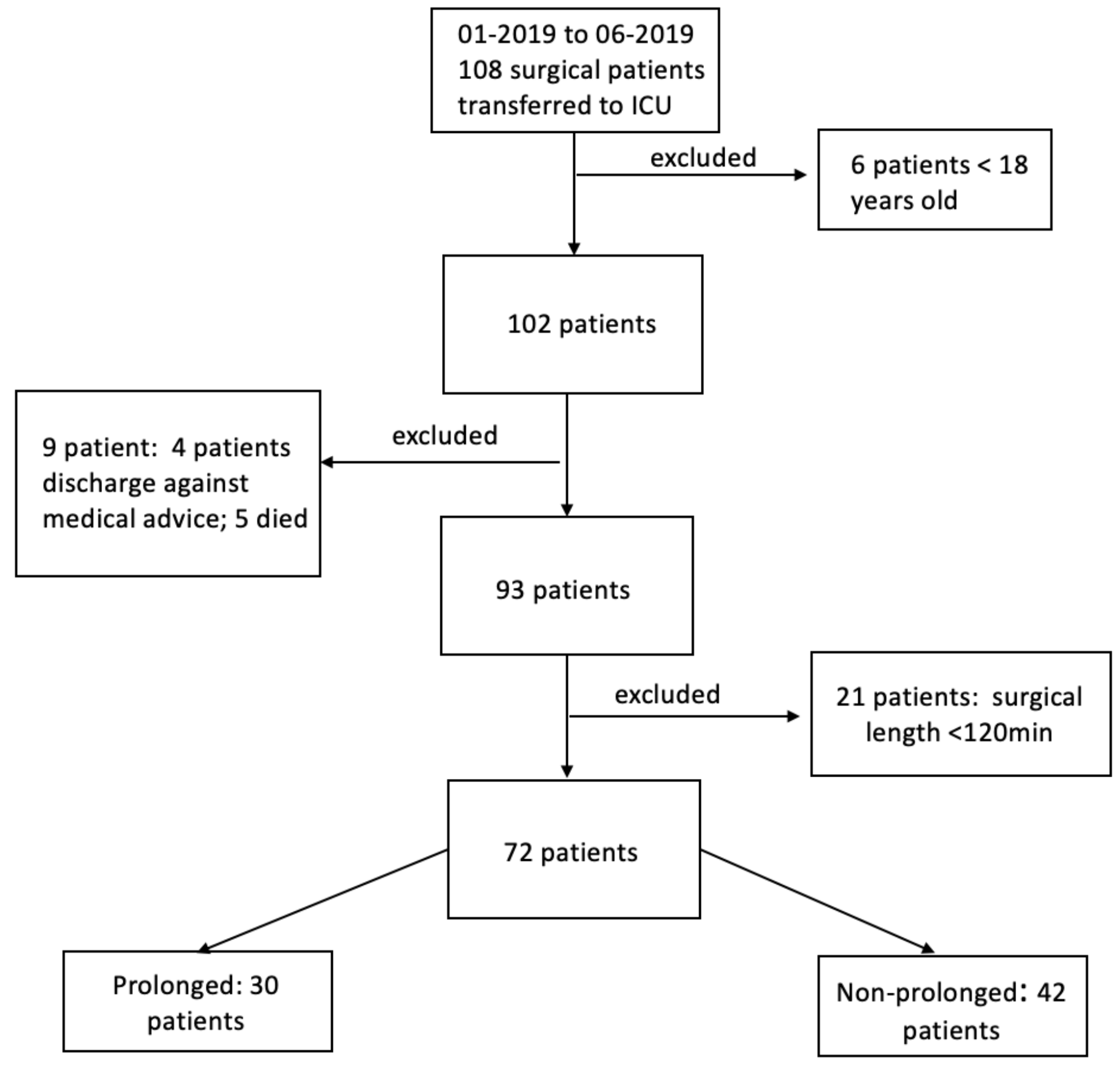

Figure 1

Diagram showing the enrollment of patients 


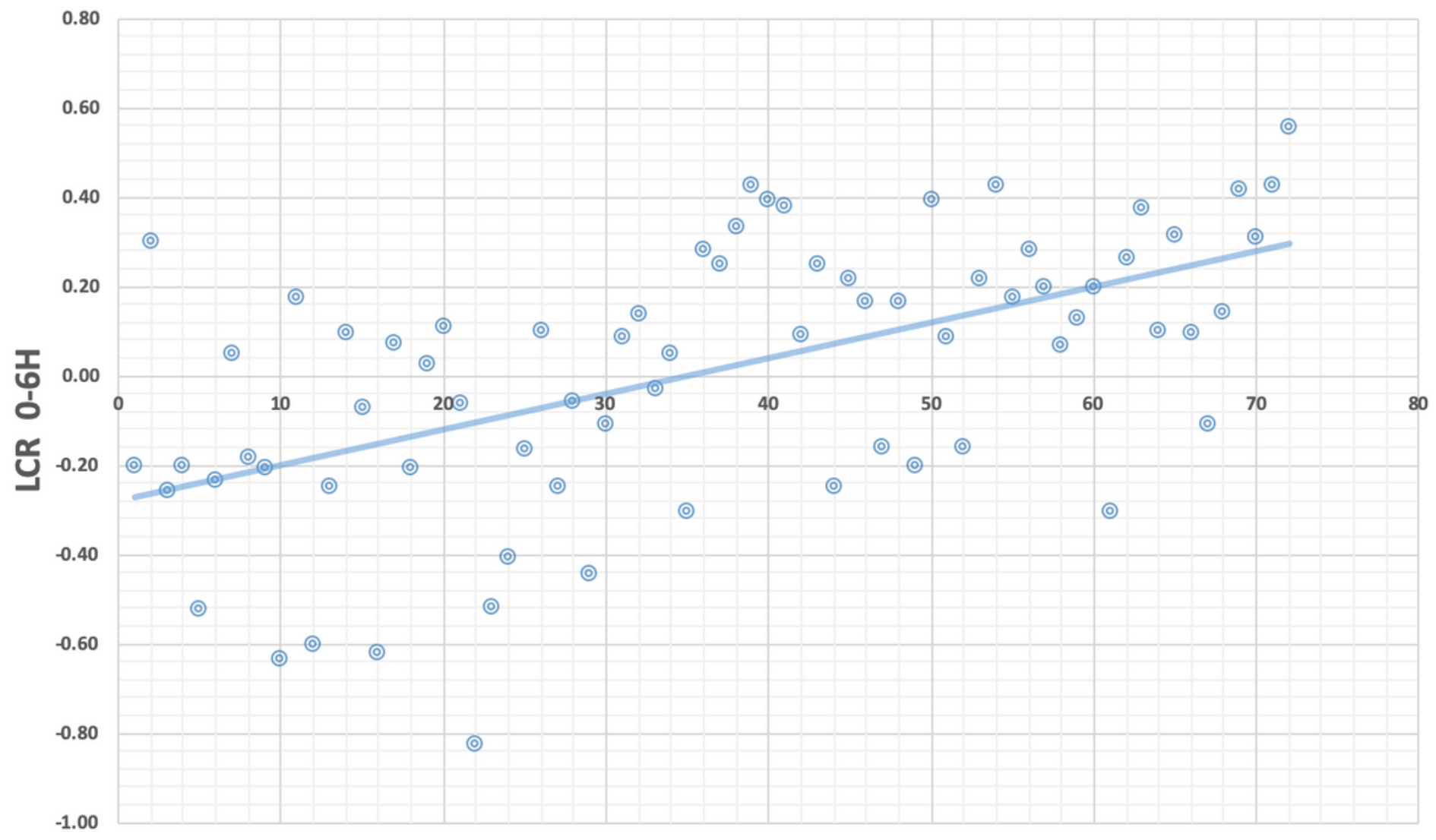

PPI 1H

Figure 2

The relationship between PPI at T0 and LCR T0-1

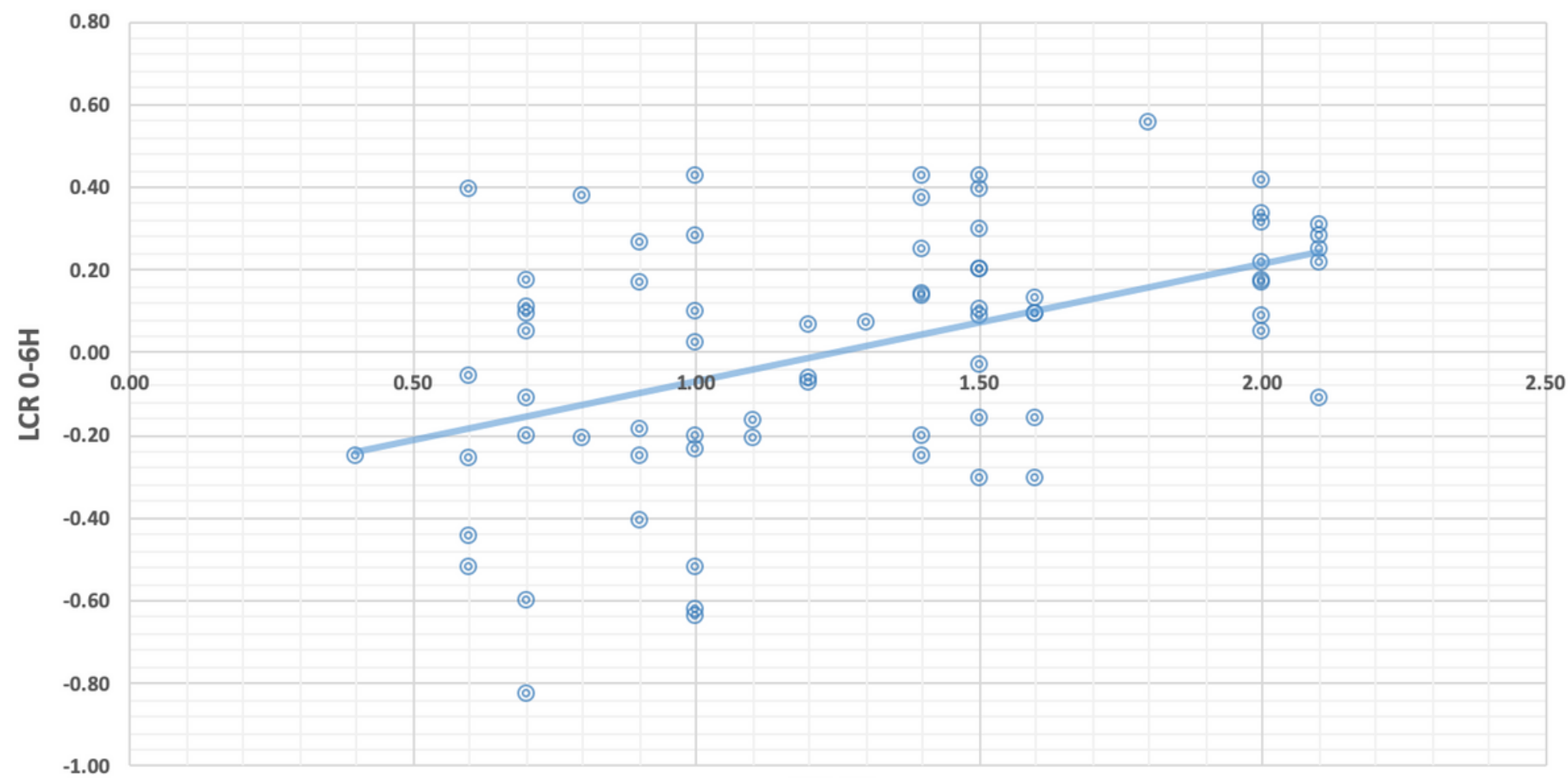

PPI 6H 
Figure 3

The relationship between PPI at T1 and LCR T0-1

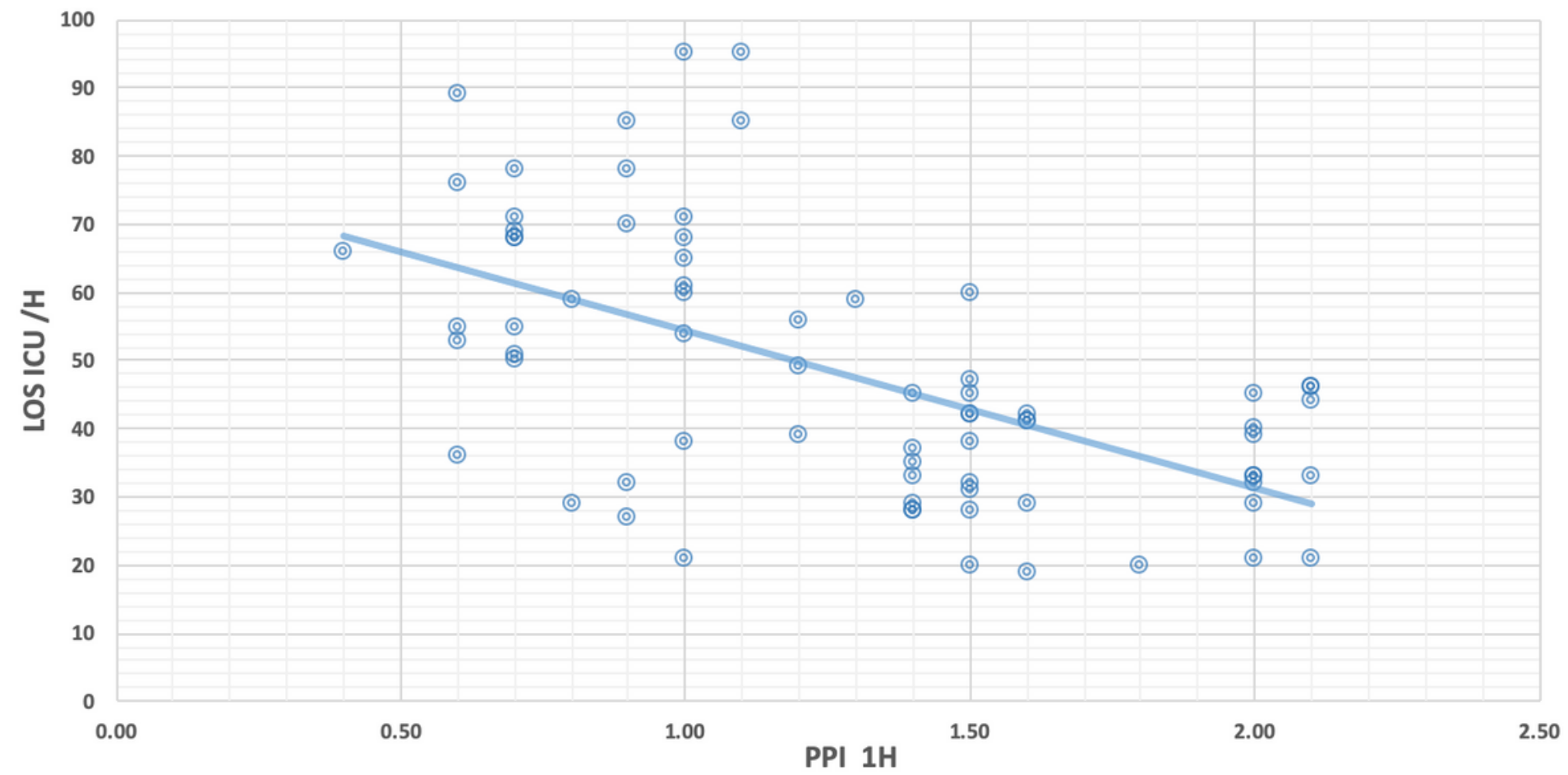

Figure 4

The relationship between PPI at TO and LOS ICU

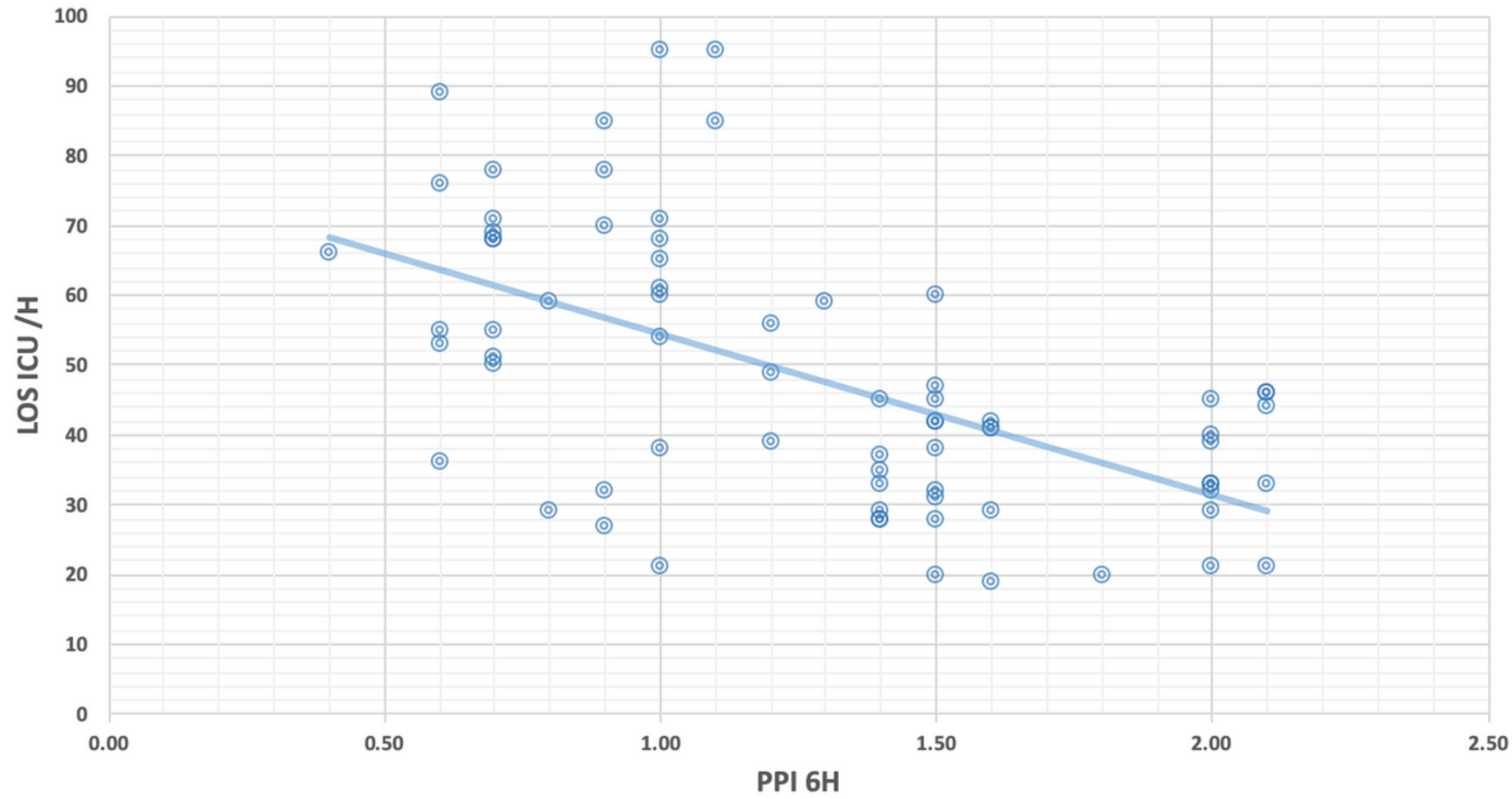

Page 15/16 
Figure 5

The relationship between PPI at T1 and LOS ICU

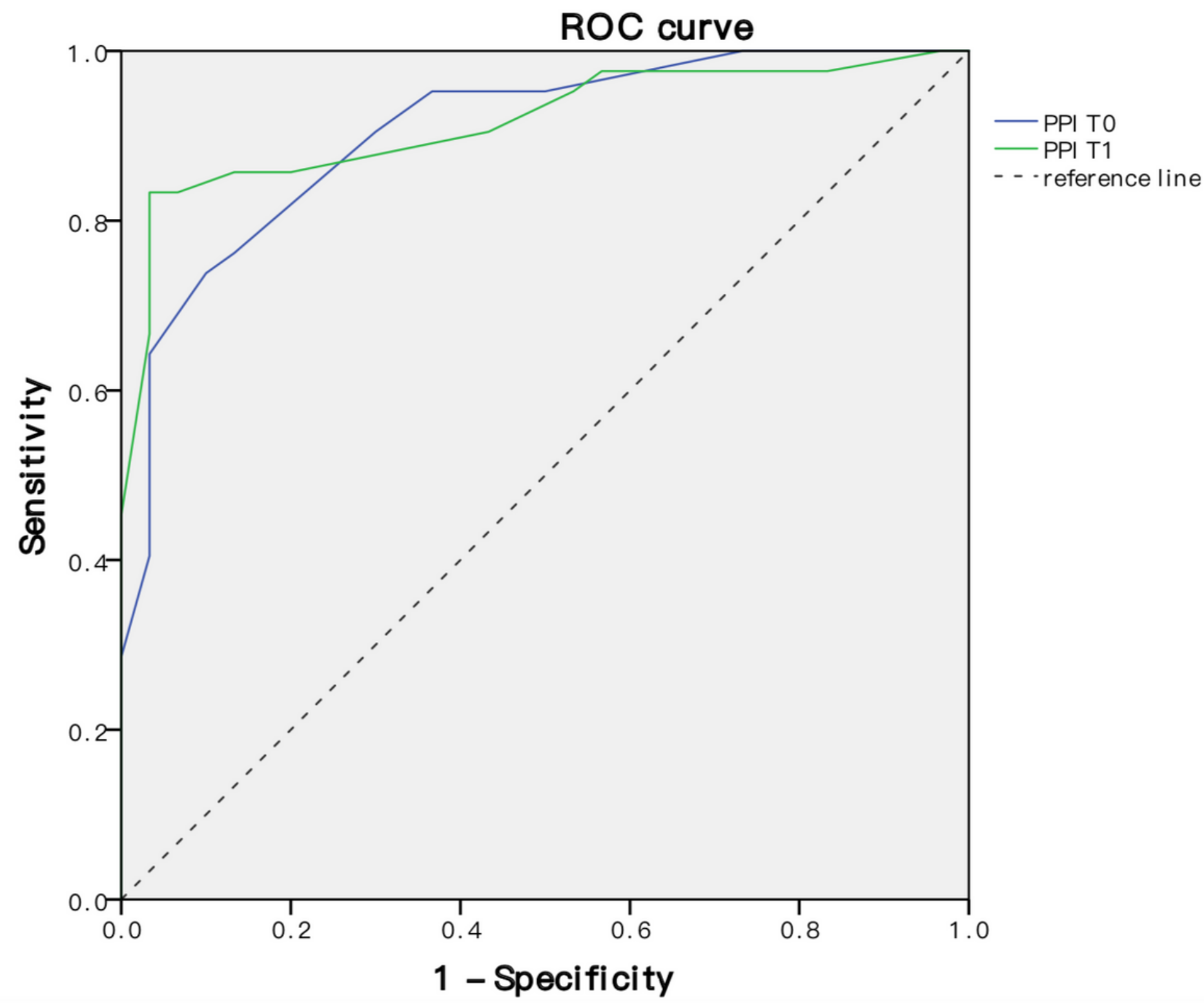

Figure 6

The ROC curves of PPI at T0 and T1 for non-prolonged ICU stay in surgical patients transferred to ICU 